\title{
Filtering methods for symmetric cardinality constraint
}

\author{
Waldemar Kocjan, Per Kreuger \\ Swedish Institute Of Computer Science \\ $\{$ waldemar, piak\}@sics.se
}

2003.08 .21

\begin{abstract}
The symmetric cardinality constraint is described in terms of variables $X=\left\{x_{1}, \ldots, x_{k}\right\}$ which take values in the subset $V=\left\{v_{1}, \ldots, v_{n}\right\}$. It constraints the number of times a value can be assigned to a variable in $X$ to be in an interval $\left[l_{x_{i}}, c_{x_{i}}\right]$ and at the same time it restricts the number of values in $V$ which any variable can take to an interval $\left[l_{v_{j}}, c_{v_{j}}\right]$. In this paper we introduce the symmetric cardinality constraint and define set constraint satisfaction problem as a framework for dealing with this type of constraints. Moreover, we present effective filtering methods for the symmetric cardinality constraint.
\end{abstract}

SICS Technical Report T2003:10

ISSN 1100-3154

ISRN:SICS-T-2003/10-SE

Keywords Constraint Programming, Global Constraints, Flow Theory

\section{Introduction}

The symmetric cardinality constraint is specified in terms of a set of variables $X=\left\{x_{1}, \ldots, x_{k}\right\}$, which take their values as subsets of $V=$ $\left\{v_{1}, \ldots, v_{n}\right\}$. The cardinality of the set assigned to each variable is constrained by the interval $\left[l_{x_{i}}, c_{x_{i}}\right]$, where $l_{x_{i}}$ and $c_{x_{i}}$ are non-negative integers. In addition, it constraints the number of occurrences of each value $v_{j} \in V$ in the sets assigned to variables in $X$ to be an interval $\left[l_{v_{j}}, c_{v_{j}}\right]$. Both $l_{v_{j}}$ and $c_{v_{j}}$ are non-negative integers.

The symmetric cardinality constraint problems arise in many real-life problems. For example, consider an instance of a project management problem. The main task of the problem is to assign personnel with possibly multiple specialized competences to a set of tasks, each requiring a certain number of people (possibly none) of each competence. In this instance we consider a project consisting of 7 activities numbered from 1 to 7, each demanding a number of persons to be accomplished. An activity which demands minimum 0 persons is optional. 


\begin{tabular}{|l|l|l|l|l|l|l|l|}
\hline & 1 & 2 & 3 & 4 & 5 & 6 & 7 \\
\hline$x_{1}$ & 1 & 1 & 0 & 0 & 0 & 0 & 0 \\
$x_{2}$ & 0 & 1 & 1 & 0 & 0 & 0 & 0 \\
$x_{3}$ & 1 & 0 & 1 & 0 & 0 & 0 & 0 \\
$x_{4}$ & 0 & 1 & 0 & 1 & 0 & 0 & 0 \\
$x_{5}$ & 0 & 0 & 1 & 1 & 1 & 0 & 0 \\
$x_{6}$ & 0 & 0 & 0 & 0 & 1 & 1 & 0 \\
\hline
\end{tabular}

\begin{tabular}{|c|c|}
\hline person & activities \\
\hline$x_{1}$ & $2 . .3$ \\
$x_{2}$ & $0 . .2$ \\
$x_{3}$ & $0 . .1$ \\
$x_{4}$ & $1 . .1$ \\
$x_{5}$ & $0 . .3$ \\
$x_{6}$ & $0 . .2$ \\
\hline
\end{tabular}

\begin{tabular}{|c|c|}
\hline task & persons \\
\hline 1 & $1 . .3$ \\
2 & $1 . .2$ \\
3 & $0 . .1$ \\
4 & $1 . .2$ \\
5 & $1 . .3$ \\
6 & $0 . .2$ \\
7 & $1 . .1$ \\
\hline
\end{tabular}

Figure 1: Project assignment specification. In the left table, a 1 indicates that a person represented by $x_{i}$ is qualified to perform corresponding activity in the project. The table in the center and to the right specifies the number of activities each person can perform and the number of persons required by each activity.

There are 6 members of personnel which can be assigned to this project. Each of those persons, here referred to as $x$ with a respective index, is qualified to perform respective activities as shown in figure 1 . A non-zero value of a lower bound indicates that members of the staff represented by the variable must be assigned to some activities in the project.

The goal is to produce an assignment which satisfy the following constraints:

- every member of staff must be assigned to a minimum and maximum number of activities in the project,

- every activity must be performed by a minimum and maximum number of persons,

- each person can be assign only to an activity he/she is qualified to perform and, by symmetry, each activity must be performed by qualified personnel.

In this paper we show how such problem can be modeled as a constraint satisfaction problem. First, in Section 2 we give some preliminaries on graphs and flows. Then, in Section 3 we define set constraint satisfaction problem and give a formal definition of the symmetric cardinality constraint. The following section, 4 , gives a method for checking consistency of symmetric cardinality constraint . Finally, we describe a filtering method for symmetric cardinality constraint.

\section{Preliminaries}

\subsection{Graph}

The following definitions are mainly due to Ahuja et al. [1993].

A directed graph $G=(X, U)$ consists of a set of nodes (vertices) $X$ and arcs (edges) $U$, where every pair $(u, v)$ is an ordered pair of distinct nodes. An oriented graph is a directed graph having no symmetric pair of arcs.

A directed network is a directed graph whose nodes and/or arcs have associated numerical values. In this paper we do not make distinction between terms "network" and "directed network". 
An $\operatorname{arc}(u, v)$ connects node $u$ with node $v$, i.e. in directed graph it is an arc oriented from node $u$ to node $v$. A path in a graph $G$ from $v_{1}$ to $v_{k}$ is a sequence of nodes $\left[v_{1}, \ldots, v_{k}\right]$ such that each $\left(v_{i}, v_{i+1}\right)$ is an arc for $i \in[1, \ldots, k-1]$. The path is simple if all its nodes are distinct. A path is a cycle if $k>1$ and $v_{1}=v_{k}$.

If a graph contains at least one path from node $i$ to node $j$ then such nodes are connected. A graph is connected if every pair of its nodes is connected, otherwise is disconnected.

The maximal connected subgraphs of a graph are referred to as its components. A component is strongly connected if it contains at least one directed path from every node to every other node.

\section{$2.2 \quad$ Flows}

Let $N$ be a directed network in which each $\operatorname{arc} e$ is associated with two non-negative integers $l(e)$ and $c(e)$ representing lower bound and a capacity of flow on $e$. A flow $f(e)$ on arc $e$ represents the amount of commodity that the arc accommodates. More formally:

Definition 2.1 A flow in a network $N$ is a function that assigns to each arc e of the network a value $f(e)$ in such way that

1. $l(e) \leq f(e) \leq c(e)$, where $l(e)$ is a lower bound of the flow in the arc and $c(e)$ is a capacity of $e$

2. for each node $n$ in the network $N$ it is true that $\sum_{n} f(e(n, p))=$ $\sum_{r} f(e(p, r))$ where $e(x, y)$ is an arc from node $x$ to $y$

The property 2 is known as a conservation law and states that the amount of flow of some commodity incoming to each node in $N$ is equal the amount of that commodity leaving each node.

Three problems from the flow theory are referred to in this paper:

- the feasible flow problem which resolves if there exists a flow in $N$ which satisfies lower bound and capacity constraints for all arcs in $N$.

- the problem of maximum flow in $N$ which finds a feasible flow in $N$, which value is maximum.

- the problem of minimum flow in $N$ which finds a feasible flow in $N$, which value is minimum.

Moreover, we refer in this paper to the residual network, which is a network representing the utilization and remaining capacity in the network with respect to flow $f$.

Definition 2.2 Given a flow $f$ from $s$ to $t$ in the network $N$, the residual network for $f$, denoted by $R(f)$, consists of the same set of nodes as $N$. The arc set of $R(f)$ is defined as follows. For all arcs $(m, n)$ in $N$

- if $f(m, n)<c(m, n)$ then $(m, n)$ is an arc of $R(f)$ with residual capacity $\operatorname{res}(m, n)=c(m, n)-f(m, n)$,

- if $f(m, n)>l(m, n)$ then $(m, n)$ is an arc of $R(f)$ with residual capacity $\operatorname{res}(m, n)=f(m, n)-l(m, n)$, 


\section{Set constraint satisfaction problem}

We define a set constraint satisfaction problem as follows.

Definition 3.1 A set constraint satisfaction problem is a triple $(X, D, C s)$ where

1. $X=\left\{x_{1}, \ldots, x_{n}\right\}$ is a finite set of (set) variables.

2. $D=\left\{D_{1}, \ldots, D_{n}\right\}$ is a set of finite sets of elements such that for each $i, x_{i}$ takes values in a subset of $D_{i}$.

3. Cs is a set of constraints on the values particular subsets of the variables in $X$ can simultaneously take. Each constraint $C \in C s$ constrains the values of a subset $X(C)=\left\{x_{C_{1}}, \ldots, x_{C_{k}}\right\}$ of the variables in $X$ and may be thought of as a subset $T(C)$ of the Cartesian product $=C_{C_{1}} \times \ldots, \times C_{C_{k}}$ where each $C_{C_{j}}=\left\{C \mid C \subseteq D_{C_{j}}\right\}$.

Let

$$
D(C)=\bigcup_{j \in\left\{j \mid x_{j} \in X(c)\right\}} D_{i}
$$

be the set of values that can be taken by any variable in $X(C)$. Furthermore, for a given assignment $P$, let $P\left(x_{i}\right)$ denote the value assigned to the variable $x_{i}$ by $P$ and \# $\left(x_{i}, P\right)$, the cardinality $\left|P\left(x_{i}\right)\right|$ of the set $P\left(x_{i}\right)$ and for any constraint $C$ and element $v_{j} \in D(C)$, \# $\left(v_{j}, C, P\right)$ denote the number of occurrences of $v_{j}$ in the values assigned by $P$ to the variables in $X(C)$, i.e:

$$
\sum_{x_{i} \in X(C)}\left\{\begin{array}{l}
1 \text { if } v_{j} \in P\left(x_{i}\right) \\
0 \text { otherwise }
\end{array}\right.
$$

Definition 3.2 A $s C S P\langle X, D, C s\rangle$ is consistent if and only if there exists an assignment $P$ with the following properties:

1. For each variable $x_{i} \in X$ with domain $D_{i}$, the value $P\left(x_{i}\right)$ assigned to $x_{i}$ by $P$ must be a subset of $D_{i}$.

2. For each constraint $C \in C$ s and each variable in $X(C)=\left\{x_{C_{1}}, \ldots, x_{C_{k}}\right\}$ the tuple $\left\langle P\left(x_{C_{1}}\right), \ldots, P\left(x_{C_{k}}\right)\right\rangle \in T(C)$.

Moreover, a value $v$ is consistent with $C$ iff $\exists P(P(X(C)) \in T(C))$ such that $v$ appears in the value $P\left(x_{i}\right)$ and $v \in P\left(X_{C_{i}}\right)$ for some $i$.

An $n$-ary constraint can be seen in terms of its value graph (Laurière [1978]), i.e the bipartite graph $G(C)=(X(C), D(C), E)$, where for all $x \in X(C), v \in D(C),\left(x_{i}, v\right) \in E$ iff $v \subseteq D_{i}$. This graph establishes an immediate correspondence between any assignment $P$ and a special set of edges in a value graph.

We formulate this notion in the following proposition.

Proposition 3.1 For any $C \in C$ s every $P(X(C))$ corresponds to a subset of edges in $G(C)$ and the number of edges connecting $x_{i} \in X(C)$ with any $v_{j} \in D(C)$ corresponds to the cardinality of the subset $P\left(x_{i}\right)$.

\section{Consistency of the symmetric cardinal- ity constraint}

We define the symmetric cardinality constraint as follows. 

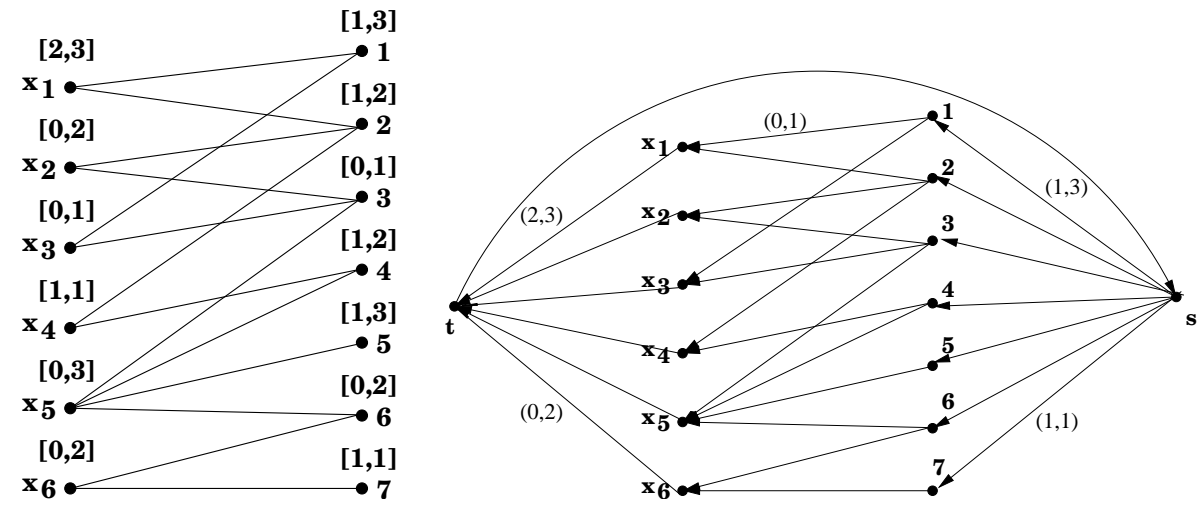

Figure 2: Value graph for assignment problem from figure 1 and its value network

Definition 4.1 A symmetric cardinality constraint is a constraint $C$ over a set of variables $X(C)$ which associate with each variable $x_{C_{i}} \in X(C)$ two non-negative integers $l_{C_{i}}$ and $u_{C_{i}}$, and with each value $v_{j} \in D(C)$ two other non-negative integers $l_{j}$ and $u_{j}$ such that a restriction of an assignment $P$ to the variables in $X(C)$ is an element in $T(C)$ iff $\forall i\left(l_{C_{i}} \leq \#\left(x_{C_{i}}, P\right) \leq u_{c_{i}}\right)$ and $\forall j\left(l_{j} \leq \#\left(v_{j}, C, P\right) \leq u_{j}\right)$.

From the symmetric cardinality constraint we propose to build a particular oriented graph, which we denote $N(C)$. This extends the value network of the global cardinality constraint as described in Régin [1996] to handle sets of nonnegative cardinality assigned to the variables. Then, we will show an equivalence between the existence of a feasible flow in such graph and the consistency of the symmetric cardinality constraint.

Let $C$ be a symmetric cardinality constraint, the value network $N(C)$ of $C$ is an oriented graph with a capacity and a lower bound on each arc. The value network $N(C)$ is obtained from the value graph $G(C)$ by

- orienting each edge of $G(C)$ from values to variables. Since each value can occur in a subset assigned to a variable at least 0 and at most 1 time for each $\operatorname{arc}(v, x): l(v, x)=0, c(v, x)=1$

- adding a source node $s$ and connecting it which each value. For every $\operatorname{arc}\left(s, v_{i}\right): l\left(s, v_{i}\right)=l_{v_{i}}, c\left(s, v_{i}\right)=c_{v_{i}}$

- adding a sink node $t$ and an arc from each variable to $t$. For each such $\operatorname{arc}\left(x_{i}, t\right): l\left(x_{i}, t\right)=l_{x_{i}}, c\left(x_{i}, t\right)=c_{x_{i}}{ }^{1}$

- adding an $\operatorname{arc}(t, s)$ with $l(t, s)=0$ and $c(t, s)=\infty$

Proposition 4.1 Let $C$ be a symmetric cardinality constraint and $N(C)$ be the value network of $C$. The following properties are equivalent:

- $C$ is consistent;

- there exists a flow from $s$ to $t$ which satisfies lower bounds and capacities of the arcs in $N(C)$.

proof: Suppose that $C$ is consistent then $T(C) \neq \emptyset$. Consider $P \in$ $T(C)$. We can build a function $f$ in $N(C)$ as follows:

\footnotetext{
${ }^{1}$ Actually, the orientation of the graph has no importance. Here, we have chosen the same orientation as in Régin [1996]
} 
1. $\forall x_{i} \in X(C), f\left(x_{c_{i}}, t\right)=\#\left(x_{c_{i}}, P\right)$

2. $\forall x_{i} \in X(C), f\left(v, x_{i}\right)=1$ if $v$ appears in the subset $P\left(x_{i}\right)$, otherwise $f\left(v, x_{i}\right)=0$

3. $\forall v_{j} \in D(C), f\left(s, v_{j}\right)=\#\left(v_{j}, C, P\right)$

Since $C$ is consistent then $\forall x_{i} \in X(C), 0 \leq l_{x_{i}} \leq \#\left(x_{i}, P\right) \leq c_{x_{i}}$ and $\forall v_{j} \in D(C), 0 \leq l_{v_{j}} \leq \#\left(v_{j}, C, P\right) \leq c_{v_{j}}$, which satisfies the lower bound and capacity constraint for the flow $f\left(v_{i}, x_{i}\right)$. Furthermore from (1)- (3) follows that $v_{j}$ must appear \# $\left(v_{j}, C, P\right)$ times in $P(X(C))$, which means that $\sum_{i=1}^{k} f\left(v_{j}, x_{i}\right)=f\left(s, v_{j}\right)$ which satisfies the conservation law for all $v_{j} \in D(C)$. From this and (2) follows that for each $x_{i} \in X(C)$ the number of arcs with flow value 1 entering $x_{i}$ is equal to \# $\left(x_{i}, P\right)$. According to (1): $\forall x_{i} \in X(C S), f\left(x_{i}, t\right)=\#\left(x_{i}, P\right)$ which satisfies the conservation law for all $x_{i} \in X$.

On the other hand, suppose there exists a feasible flow $f$ from $s$ to $t$. Since $f\left(v_{j}, x_{i}\right)=1$ or $f\left(v_{j}, x_{i}\right)=0$ and by the conservation law $f\left(s, v_{j}\right)=$ $\sum_{i=1}^{k} f\left(v_{j}, x_{i}\right)$ then for each $v_{j}$ the number of edges with the flow of value 1 leaving $v_{j}$ is equal $f\left(s, v_{j}\right)$. Consequently, the number of $x_{i} \in X(C)$ connected with each $v_{j}$ by an arc with a flow equal to 1 is equal to $f\left(s, v_{j}\right)$. Furthermore, due to the conservation law, the number of arcs for which $f\left(v_{j}, x_{i}\right)=1$ entering each $x_{i}$ is equal the value of $f\left(x_{i}, t\right)$. Thus the set of arcs such that $f\left(v_{j}, x_{i}\right)=1$ corresponds to a set of edges in the value graph. By the capacity constraint $\forall v_{i} \in D(C): l_{v_{i}} \leq f\left(s, v_{i}\right) \leq$ $c_{v_{i}}$ and by the conservation law $f\left(s, v_{i}\right)=\sum_{x} f\left(v_{i}, x\right) \leq c_{i}$ therefore $l_{i} \leq \#\left(v_{i}, C, P\right) \leq c_{i}$. Similarly, $\forall x_{i} \in X: l_{c_{x_{i}}} \leq \sum_{i=1}^{k} f\left(v_{j}, x_{i}\right) \leq c_{x_{i}}$ and by the conservation law $f\left(x_{i}, t\right)=\sum f\left(v_{j}, x_{i}\right) \leq c_{x_{i}}$, therefore $l_{x_{i}} \leq$ $\#\left(x_{i}, P\right) \leq c_{x_{i}}$, thus $C$ is consistent.

This proposition gives a way of checking the consistency of a symmetric cardinality constraint by computing a feasible flow in $N(C)$. Different algorithms for computing feasible flows are given in the literature on flow theory, e.g. in Ahuja et al. [1993, 1997].

In the next section we will show how to implement filtering of symmetric cardinality constraint by considering certain properties common to all feasible flows in the value graph.

\section{Filtering algorithm for symmetric car- dinality constraint}

Let $f$ be a feasible flow from $m$ to $n$ in a network $N, R(f)$ be a residual graph for $f$ and $\operatorname{res}(p)$ be a residual capacity of a simple path $p$ in $R(f)$, where $\operatorname{res}(p)$ is a minimum value of the residual capacity of each arc in $p$. If there is a simple path $p$ from $m$ to $n$, then we can obtain a new feasible flow $f^{\prime}(n, m)=f(n, m)+\min (\operatorname{res}(p)$, res $(m, n))$. We call such a $p$ an augmenting path. Similarly, if there exists a simple path $p$ from $m$ to $n$, which does not contain $(m, n)$ and if $f(n, m)>l(n, m)$ then we obtain a new feasible flow $f^{\prime}(n, m)=f(n, m)-\min (\operatorname{res}(p), \operatorname{res}(n, m))$. We refer to such simple path $p$ as a reducing path.

Moreover, the maximum and minimum flow are defined as follows (Régin [1996]).

Definition 5.1 A flow $f$ from $m$ to $n$ is maximum if and only if there is no augmenting path from $m$ to $n$ for $f$. 
A flow $f$ from $m$ to $n$ is minimum if and only if there is no reducing path from $m$ to $n$ for $f$.

The following theorem 5.1 gives a way of determining if an arbitrary arc in $N$ is contained in any feasible flow in the network. The theorem is similar to Theorem 4 from Régin [1996], but here the computation is performed on the residual graph of $f$ which includes both $(t, s)$ and, in the case when $f(t, s)>0$, also $(s, t)$.

Theorem 5.1 Let $N$ be a network for which each arc is associated with two non-negative integers, $f$ be an arbitrary feasible flow in $N, R(f)$ be the residual graph for $f$ and $(m, n)$ be an arbitrary arc in $N$. For all feasible flows $f^{\prime}$ in $N, f^{\prime}(m, n)=f(m, n)$ if and only if neither $(m, n)$ nor $(n, m)$ are contained in any simple cycle in $R(f)$ involving at least three vertices.

Proof: If $(m, n)$ is not contained in a simple cycle in $R(f)$ involving at least three vertices it means that there is no augmenting path from $n$ to $m$ for $f$. By the definition 5.1, the flow $f$ is the maximum flow from $m$ to $n$.

If $(n, m)$ is not contained in a simple cycle in $R(f)$ which involves at least three nodes then there is no reducing path from $n$ to $m$ in $N$, so by the definition $5.1 f$ is the minimum flow from $m$ to $n$.

Similarly, if $(t, s)$ is not contained in a simple cycle in $R(f)$ with at least 3 nodes, then there is no augmenting path from $s$ to $t$ and by definition 5.1 $f$ is a maximum flow in $N$. Moreover, if $(s, t)$ is not contained in a simple cycle in $R(f)$ involving at least three nodes then there is no reducing path for $f$ in $R(f)$ and by definition $5.1 f$ is a minimum flow in $N$.

Let $C \in C s$ be a symmetric cardinality constraint and $f$ be an arbitrary feasible flow in $N(C)$. By Proposition 4.1 a value $v$ of a variable $x$ is not consistent in $C$ if and only if there exists no feasible flow in $N(C)$ which contains $(v, x)$. So, by the Theorem 5.1 if $f(v, x)=0$ and $(v, x)$ is not contained in a simple cycle in $R(f)$ involving at least three nodes then the value $v$ of a variable $x$ is not consistent with $C$. Since the nodes connected by any arc $(m, n)$ must belong to the same strongly connected component we can compute if any value $v$ of variable $x$ such that $f(v, x)=0$ is consistent with $C$ using the algorithm which searches for strongly connected components in a graph, e.g. the algorithm described in [Tarjan, 1972].

This leads to the following corollary:

Corollary 5.1 The consistency of any value $v$ of variable $x$ with symmetric cardinality constraint $C$ can be computed by finding an arbitrary feasible flow $f$ from $s$ to $t$ in $N(C)$ and by computing strongly connected components in $R(f)$.

\section{Conclusion}

In this paper we have introduced the symmetric cardinality constraint derived from the global cardinality constraint. Moreover, we have formalized set constraint satisfaction problem and defined symmetric cardinality constraint in the context of this problem. We have also presented efficient methods for filtering domains of symmetric cardinality constraint. 


\section{Acknowledgment}

The authors wish to thank Nicolas Beldiceanu, Irit Katriel and Markus Bohlin for their helpful comments on this paper.

\section{References}

R. Ahuja, M. Kodialam, A. Mishra, and J. Orlin. Computational investigations of maximum flow algorithms. European Journal of Operational Research, 97(3):509-542, 1997.

R. Ahuja, T. Magnanti, and J. Orlin. Network flows. Theory, algorithms and applications. Prentice-Hall Inc., 1993.

J.-L. Laurière. A language and a program for stating and solving combinatorial problems. Artificial Intelligence, 10:29-127, 1978.

J.-C. Régin. Generalized arc consistency for global cardinality constraint. In Proc. of the Fourteenth National Conference on Artificial Intelligence (AAAI-96), 1996.

R. Tarjan. Depth-first search and linear graph algorithms. SIAM J. Computing, 1(2):146-160, June 1972. 